\title{
Overexpression of goosecoid homeobox is associated with chemoresistance and poor prognosis in ovarian carcinoma
}

\author{
KYONG-WON KANG ${ }^{1}$, MI-JUNG LEE ${ }^{1,2}$, JI-AE SONG ${ }^{1}$, JU-YEON JEONG ${ }^{1}$, YOO-KYONG KIM ${ }^{1}$, \\ CHAN LEE ${ }^{3}$, TAE-HEON KIM ${ }^{1,2}$, KYU-BEOM KWAK ${ }^{4}$, OK-JUN KIM ${ }^{1}$ and HEE JUNG AN ${ }^{1,2}$ \\ ${ }^{1}$ Institute for Clinical Research, College of Medicine, CHA University; Departments of ${ }^{2}$ Pathology and \\ ${ }^{3}$ Gynecologic Oncology, College of Medicine, CHA University; ${ }^{4}$ Department of Biomedical Science, \\ College of Life Science, CHA University, Sungnam 463-712, Republic of Korea
}

Received March 11, 2014; Accepted April 29, 2014

DOI: $10.3892 /$ or.2014.3203

\begin{abstract}
Ovarian carcinoma is the most lethal cancer among all gynecological malignancies due to recurrence through chemoresistance. The aim of the present study was to identify new biomarkers to predict chemoresistance and prognosis in ovarian carcinomas. The mRNA expression by qRT-PCR was examined in 60 ovarian serous carcinomas for selected genes from the screening by PCR array focusing on apoptosis, epithelial-to-mesenchymal transition and cancer pathways. The clinical impact was assessed by analyzing the correlation between gene expression and clinicopathological variables. Further validation with immunohistochemistry was performed with 75 cases of serous carcinomas. The chemoresistance was significantly associated with high expression of FOS, GSC, SNAI1, TERT and TNFRSF10D, and low expression of CDKN1A, TNFRSF10A, TNFRSF10C and TRAF1 $(\mathrm{P}<0.05$, t-test). Low expression of TRAF1 and high expression of E2F1, FOS, TERT and GSC were significantly associated with advanced clinical stage $\left(\mathrm{P}<0.05, \chi^{2}\right.$-test). Lymph node metastasis was significantly associated with high expression of GSC. The upregulation group of TERT, GSC, NOTCH1 and SNAI1, and downregulation group of TRAF1 were significantly associated with poor overall survival $(\mathrm{P}<0.05$, log-rank test). On further validation with immunohistochemistry, overexpression of goosecoid homeobox (GSC) was associated with poor overall survival. The results suggest that GSC is the most potential biomarker of drug response and poor prognosis in ovarian serous carcinomas.
\end{abstract}

Correspondence to: Professor Hee Jung An or Professor Ok-Jun Kim, Institute for Clinical Research, College of Medicine, CHA University, 351 Yatap-dong, Bundang-gu, Seongnam-si, Gyeonggi-do 463-712, Republic of Korea

E-mail: hjahn@cha.ac.kr

E-mail:ercir@cha.ac.kr

Key words: ovarian carcinoma, chemoresistance, PCR array, qRT-PCR, immunohistochemistry

\section{Introduction}

Ovarian carcinoma is the most lethal cancer among all gynecological malignancies (1) due to late detection and recurrence through chemoresistance. Despite debulking surgery and response to first-line chemotherapy with platinum and Taxol, the majority of patients, up to $75 \%$, eventually suffer from recurrence due to chemoresistance (2), and $90 \%$ of the deaths from ovarian cancer can be attributed to chemoresistance.

Ovarian carcinoma is a heterogeneous group of neoplasms and comprises several histological subtypes: serous, mucinous, endometrioid, clear cell and undifferentiated carcinomas. The present study focused on high-grade serous carcinoma, which accounts for $80 \%$ of epithelial ovarian carcinoma cases and has the highest recurrence rate. Beyond the knowledge that a high incidence of molecular alterations targeting TP53, HER-2 and PIK3CA $(3,4)$ is associated with high-grade serous carcinoma, relatively little is known concerning the molecular alterations associated with recurrence and chemoresistance. Therefore, a better understanding of the molecular mechanisms leading to chemoresistance may provide new biomarkers for predicting patient prognosis and survival, and new treatment strategies for life-threatening ovarian cancer $(5,6)$.

Previous studies have shown that chemoresistance is multifactorial, involving drug inactivation/efflux, microtubule regulation (7), increased DNA repair, alterations in cell cycle control, changes in apoptotic threshold and signaling pathways such as the PI3K/AKT pathway (8). Recently, new evidence suggests that epithelial-to-mesenchymal transition (EMT) plays a significant role in chemoresistance in various human cancers $(9,10)$. In addition, it was demonstrated that EMT transcription factors, snail and slug, contribute to cisplatinresistance and cancer metastasis in ovarian cancer $(11,12)$. However, we did not find any comprehensive gene expression study focusing on EMT in ovarian cancer.

In the present study, the gene expression of clinical specimens of high-grade ovarian serous carcinoma was examined using qRT-PCR after screening with PCR arrays focusing on apoptosis, EMT and cancer pathways to understand the molecular mechanism of chemoresistance and to identify novel biomarkers to predict the patient outcome. The mRNA expression levels of genes, which were significantly altered in 
the chemoresistant tumors compared to those of the chemosensitive tumors, were correlated with clinicopathological parameters, including stage, metastasis (lymph node and distant) and survival to evaluate the clinical impact. Our results demonstrated that high expression of TERT and goosecoid homeobox (GSC), and low expression of TRAF1 were associated with advanced stage, chemoresistance and poor overall survival. Further validation with immunohistochemistry confirmed that GSC overexpression was correlated with poor overall survival.

\section{Materials and methods}

Patients and clinical specimens. For the PCR array and qRT-PCR to evaluate mRNA expression, 60 fresh tissues from high-grade ovarian serous carcinomas were obtained at the time of surgery from patients undergoing oophorectomies for ovarian carcinomas at the Bundang CHA Medical Center (Table I). Samples were immediately frozen in liquid nitrogen and stored at $-80^{\circ} \mathrm{C}$. Frozen sections were assessed to confirm that tumor cells represented nearly $80 \%$ of the tissue. As a normal control, 10 normal fallopian tubes were used from the patients who underwent hysterectomies for leiomyomas.

For immunohistochemical analysis, we utilized formalinfixed, paraffin-embedded ovarian epithelial tumor tissues from 75 patients with high-grade ovarian serous carcinomas who had been surgically treated at the CHA Bundang Medical Center from 1998 to 2010. Clinical and pathological data were retrieved from clinical databases and from the original pathology reports. The histologic type of the tumor was classified according to the WHO ovarian tumor classification. Tumor staging was carried out according to the tumor-nodemetastasis (TNM) staging system. The samples were divided into two groups according to responsiveness to first-line chemotherapy. Based on the NCCN guidelines, chemoresistant tumors were defined as those leading to persistent or recurrent disease within 6 months after the initiation of first-line Taxolplatinum-based combination chemotherapy. Chemosensitive tumors were classified as those with a complete response to chemotherapy and a platinum-free interval of $>6$ months. The present study was approved by the Ethics Committee of the Bundang CHA Medical Center, and informed consent was obtained from each patient prior to surgery.

PCR array. For screening the genes associated with chemoresistance, PCR arrays (SABioscience, Frederick, MD, USA) were selected that included genes involved in apoptosis (http:// sabiosciences.com/rt_pcr_product/HTML/PAHS-012Z.html), known cancer pathways (http://sabiosciences.com/rt_pcr_ product/HTML/PAHS-033Z.html) and EMT (http:// sabiosciences.com/rt_pcr_product/HTML/PAHS-090Z. html). The PCR arrays were performed using randomly selected 18 ovarian cancer specimens (13 chemosensitive and 5 chemoresistant carcinomas) among the samples enrolled in the present study. Two normal fallopian tubes were used as a control. Total RNA was isolated using TRIzol reagent (Invitrogen, Carlsbad, CA, USA) according to the manufacturer's instructions.

For first strand cDNA synthesis, $1 \mu \mathrm{g}$ total RNA was reverse-transcribed in a final reaction volume of $20 \mu \mathrm{l}$ using
Table I. Clinicopathological characteristics of the ovarian serous carcinoma patients $(n=60)$.

\begin{tabular}{lcc}
\hline & $\begin{array}{c}\text { Chemosensitive } \\
\mathrm{n}(\%)\end{array}$ & $\begin{array}{c}\text { Chemoresistant } \\
\mathrm{n}(\%)\end{array}$ \\
\hline $\begin{array}{l}\text { Mean age (years) } \\
\text { Stage }\end{array}$ & $55.5 \pm 22.5$ & $63 \pm 20$ \\
I/II & $12(20.00)$ & $1(1.67)$ \\
III/IV & $31(51.67)$ & $16(26.67)$ \\
LN status & & \\
Negative & $20(33.33)$ & $6(10.00)$ \\
Positive & $23(38.33)$ & $11(18.33)$ \\
Metastasis & & $14(23.33)$ \\
Negative & $32(53.33)$ & $3(5.00)$ \\
Positive & $11(18.33)$ & $17(28.33)$ \\
Total & $43(71.67)$ & \\
\hline
\end{tabular}

LN, lymph node.

the RT2 First Strand kit (SABioscience) according to the manufacturer's instructions. qRT-PCR was conducted on a CFX96 thermal cycler (Bio-Rad Applied Science, Mannheim, Germany) using universal cycling conditions $\left(10 \mathrm{~min}\right.$ at $95^{\circ} \mathrm{C}$, $15 \mathrm{sec}$ at $95^{\circ} \mathrm{C}, 1 \mathrm{~min} 60^{\circ} \mathrm{C}$ for 40 cycles).

For data normalization and analysis, 5 endogenous control genes, $\beta$-2-microglobulin (B2M), hypoxanthine phosphoribosyltransferase (HPRT1), ribosomal protein L13a (RPL13A), glyceraldehyde-3-phosphate dehydrogenase (GAPDH) and $\beta$-actin (ACTB) on the PCR array were used. Each replicate cycle threshold $(\mathrm{Ct})$ was normalized to the average $\mathrm{Ct}$ of 5 endogenous controls on a per plate basis. The comparative $\mathrm{Ct}$ method was used to calculate the relative quantification of gene expression. The following formula was used to calculate the relative amount of the transcripts in the treated samples and the control group, both of which were normalized to the endogenous controls. $\Delta \Delta \mathrm{Ct}=\Delta \mathrm{Ct}$ (treated) $-\Delta \mathrm{Ct}$ (control) for RNA samples. $\Delta \mathrm{Ct}$ is the $\log _{2}$ difference in $\mathrm{Ct}$ between the target gene and endogenous controls by subtracting the average $\mathrm{Ct}$ of the controls from each replicate. The fold change for each treated sample relative to the control sample $=2^{-\Delta \Delta \mathrm{Ct}}$.

PCR array quantification was based on the $\mathrm{Ct}$ number. $\mathrm{Ct}$ was defined as 35 for the $\Delta \mathrm{Ct}$ calculation when the signal was under detectable limits. A list of differentially expressed genes was identified using a two-tailed t-test. Changes in gene expression between treated groups and control groups were illustrated as a fold increase/decrease. The criteria included a $\mathrm{P}$-value $<0.05$ and a mean difference of $\geq 2$-fold. Statistical evaluation was conducted with the web-based RT2 Profiler ${ }^{\mathrm{TM}}$ PCR Array Data Analysis software (SABioscience).

Real-time RT-PCR. For the genes which were significantly altered by $>2$-fold in the PCR arrays in the chemoresistant compared to the chemosensitive tumors, real-time RT-PCR was conducted with 60 samples of serous carcinomas on a CFX96 ${ }^{\mathrm{TM}}$ real-time PCR system (Bio-Rad Applied Science). The final volume of $20 \mu \mathrm{l}$ included $0.5 \mu \mathrm{l}$ of cDNA template, 


\begin{tabular}{|c|c|c|c|c|c|c|c|c|c|c|c|c|c|c|c|c|c|c|c|}
\hline & \multirow[b]{2}{*}{ CDKN1A } & \multicolumn{13}{|c|}{ Chemosensitive } & \multicolumn{5}{|c|}{ chemoresistant } \\
\hline & & 0.188 & 0.018 & 0.052 & 0.025 & 0.017 & 0.065 & 0.022 & 0.065 & 0.066 & 0.027 & 0.053 & 0.018 & 0.073 & 0.127 & 0.462 & 0.093 & 0.036 & 0.062 \\
\hline \multirow{7}{*}{$\begin{array}{l}\text { Cancer } \\
\text { pathway }\end{array}$} & E2F1 & 5.014 & 4.601 & 3.091 & 5.586 & 6.442 & 9.317 & 5.540 & 17.377 & 10.478 & 7.888 & 14.530 & 9.802 & 24.525 & 42,405 & 26180 & 7.080 & 3.088 & 22.515 \\
\hline & FOS & 2.457 & 0.049 & 0.592 & 0.114 & 0.236 & 0.723 & 0.096 & 1.299 & 0.881 & 1.322 & 0.227 & 0.495 & 2.563 & 4.432 & 3.739 & 2.419 & 0.574 & 3.645 \\
\hline & ITGB1 & 0.065 & 0.174 & 0.269 & 0.080 & 0.075 & 0.102 & 0.074 & 0.178 & 0.189 & 0.067 & 0.154 & 0.099 & 0.165 & 0.286 & 0.107 & 0.365 & 0.456 & 0.129 \\
\hline & ITGBS & 0.171 & 0.108 & 0.154 & 0.113 & 0.280 & 0.243 & 0.044 & 0.213 & 0.329 & 0.142 & 0.183 & 0.159 & 0.106 & 0.182 & 1.038 & 0.618 & 0.238 & 0.688 \\
\hline & SERPINE1 & 002 & 0.00 & 0.001 & 0.001 & 0.001 & 0.001 & 0.00 & 0.003 & 0.001 & 0.003 & 0,001 & 0.001 & 0.017 & 0.030 & 0.070 & 0.032 & 0.005 & 0.029 \\
\hline & TERT & 3.881 & 1.584 & 0.506 & 2.618 & 0.918 & 0.205 & 0.067 & 1.041 & 0.922 & 1.880 & 2.642 & 0.814 & 3.935 & 6.804 & 2.077 & 4.319 & 7.566 & 5.388 \\
\hline & FADD & 0.033 & 0.003 & 0.004 & 0.035 & 0.131 & 0.008 & 0.035 & 0.031 & 0.076 & 0.072 & 0.029 & 0.028 & 1.102 & 1.905 & 3.732 & 1.778 & 0.511 & 0.824 \\
\hline \multirow{6}{*}{ Apoptosis } & TNFRSF10D & 1.919 & 0.237 & 0.354 & 0.200 & 0.451 & 1.765 & 0.094 & 0.178 & 0.490 & 0.291 & 0.486 & 0.222 & 0.460 & 4.532 & 0.633 & 0.291 & 4.377 & 9.126 \\
\hline & TNFRSF1A & 0.255 & 1.110 & 0.060 & 0.178 & 0.607 & 0.019 & 0.012 & 0.068 & 1.021 & 2.412 & 1.087 & 1.133 & 10.778 & 6.543 & 0.344 & 2.497 & 1.231 & 1.729 \\
\hline & TNFRSF8 & 1.464 & 0.993 & 0.986 & 1.866 & 1.765 & 0.507 & 0.312 & 0.354 & 1.986 & 4.377 & 0.940 & 1.558 & 3.784 & 1.404 & 5.897 & 1.257 & 2.412 & 15.032 \\
\hline & FN1 & 0.011 & 0.007 & 0.069 & 0.018 & 0.010 & 0.022 & 0.012 & 0.042 & 0.131 & 0.039 & 0.106 & 0,004 & 0.016 & 0.107 & 0.230 & 0.140 & 0.134 & 0.258 \\
\hline & GSC & 5.564 & 5.140 & 0.638 & 19.086 & 4.925 & 1.003 & 0.120 & 2.721 & 5.819 & 2.920 & 1.246 & 0.938 & 1.484 & 16.087 & 17.622 & 42.088 & 9.206 & 8,421 \\
\hline & NOTCH1 & 1.586 & 0.584 & 0.618 & 2.567 & 0.393 & 1.018 & 0.135 & 2.474 & 1.193 & 0.730 & 0.861 & 0.447 & 0.903 & 3.343 & 5.634 & 3.953 & 1.915 & 7.377 \\
\hline \multirow[t]{4}{*}{ EMT } & SMAD2 & 0.843 & 0.857 & 0.751 & 0.737 & 1.137 & 1.869 & 0.624 & 1.175 & 1.529 & 0.476 & 1.267 & 1.708 & 0.307 & 2.123 & 3.191 & 2.198 & 0.812 & 2.093 \\
\hline & SNAI1 & 1.779 & 0.402 & 0.277 & 0.892 & 0.234 & 0.476 & 0.101 & 1.190 & 0.495 & 0.616 & 0.698 & 0.309 & 0.972 & 1.561 & 2.991 & 4.794 & 1.736 & 8.413 \\
\hline & sox 10 & 2.674 & 1.891 & 2.901 & 12.430 & 0.451 & 15.371 & 0.154 & 3.355 & 3.752 & 1.756 & 2.286 & 2.330 & 12.537 & 14681 & 32.947 & 25.357 & 13.408 & 45.696 \\
\hline & TMEM132A & 0.144 & 0.179 & 0.215 & 0.073 & 0.088 & 0.219 & 0.019 & 0.234 & 0.626 & 0.297 & 0.201 & 0.163 & 0.136 & 0.437 & 1.474 & 0.415 & 0.496 & 0.761 \\
\hline
\end{tabular}

Figure 1. Heat map showing the expression of the significantly different genes by PCR array between the chemosensitive and chemoresistant group. Each row of the heat map represents a gene while each column represents the clinical samples (groups are labeled at the top). The color saturation scale, shown to the right, represents the level of gene expression relative to normal controls. Red indicates an increase in gene expression, whereas green indicates a decrease. Only genes whose expression demonstrated a 2.0-fold or greater increase or decrease in the chemoresistant tumors compared to the chemosensitive tumors are depicted. The list of genes comprising the heat map is summarized in Tables II-IV.

$10 \mu \mathrm{l}$ of TaqMan Master Mix (Applied Biosystems, Foster City, CA, USA), and $1 \mu \mathrm{l}$ of a mix containing primers and probes. The amplification began with $2 \mathrm{~min}$ at $50^{\circ} \mathrm{C}$, and $10 \mathrm{~min}$ at $95^{\circ} \mathrm{C}$, followed by 50 cycles of $95^{\circ} \mathrm{C}$ for $15 \mathrm{sec}$ and $60^{\circ} \mathrm{C}$ for $1 \mathrm{~min}$. The CFX Manager ${ }^{\mathrm{TM}}$ software (version 1.0) was used to determine the cycle threshold $(\mathrm{Ct})$ by default values. Individual PCRs were performed in triplicate, and the mRNA expression level of each gene relative to GAPDH was calculated using the $2^{-\Delta \Delta \mathrm{Ct}}$ method.

Tissue microarray and immunohistochemical analysis. For the validation of putative markers which showed significant association between mRNA expression and more than 2 clinicopathological parameters and survival, we performed immunohistochemical analysis with a tissue microarray (TMA) containing 75 ovarian serous carcinomas and 20 ovarian serous cystadenomas and 10 normal fallopian tubes (as a control). All tissue samples were retrieved from the archival files of the CHA Bundang Medical Center, School of Medicine, CHA University. The hematoxylin and eosin (H\&E) sections of the selected cases were reviewed, and the representative areas were marked on the H\&E-stained sections and the corresponding paraffin blocks. For each case, three tissue cores with diameters of $2 \mathrm{~mm}$ were punched out from the marked tissue areas of each donor tissue block. They were then arranged into recipient paraffin blocks using a manual microarray device (UNITMA, Quick-Ray ${ }^{\mathrm{TM}}$; Unitech Science Co., Ltd., Seoul, Korea).

Tissue microarray paraffin sections were deparaffinized in xylene for $30 \mathrm{~min}$ and rehydrated in a graded series of alcohols. Endogenous peroxidase activity was blocked via 30 min of treatment with $0.3 \%$ hydrogen peroxide in methanol solution. For antigen retrieval, the sections were heated in $0.1 \mathrm{~mol} / \mathrm{l}$ of citrate buffer ( $\mathrm{pH} 6.0$ ) for $15 \mathrm{~min}$ in a microwave oven. Slides were incubated overnight at $4^{\circ} \mathrm{C}$ with the following primary antibodies and working dilutions: TRAF1 (1:2,000; Novus Biologicals, Littleton, CO, USA), TERT (1:200; Novus Biologicals) and GSC (1:200; Abnova, Taipei, Taiwan). Thereafter, 30 min of incubation with the secondary antibody was carried out using a Dako EnVision Rabbit/Mouse kit at room temperature. The sections were then developed with diaminobenzidine and counterstained with hematoxylin. Positive nuclear or cytoplasmic staining in $>50 \%$ of the tumor cells was considered indicative of overexpression.

Statistical analysis. Significant differences between groups were determined using the Student's t-test and $\chi^{2}$-test. Survival curves were estimated using the Kaplan-Meier method and compared using the log-rank test. A P-value $<0.05$ was considered statistically significant. Statistical analysis was performed using SPSS 19.0 software (SPSS, Inc., Chicago, IL, USA).

\section{Results}

Gene expression profile by PCR array. To select candidate prognostic markers, we analyzed the mRNA expression of 18 ovarian serous carcinomas using PCR arrays including genes involved in known cancer pathways, apoptosis and EMT, and we compared the expression profile of the chemoresistant tumors to that of the chemosensitive tumors (Fig. 1). Among 84 genes involved in cancer pathways associated with cancer adhesion, apoptosis, cell senescence, cell cycle, invasion and metastasis, 6 genes, ITGB1, ITGB5, TERT, E2F1, SERPINE1 and FOS, were significantly upregulated by $>2$-fold in the chemoresistant compared to the chemosensitive tumors (Table II). CDKN1A was significantly downregulated by $>2$-fold in the chemoresistant group. Among these genes, FOS (5.76-fold), TERT (4.23-fold) and SERPINE1 (9.49-fold) had the highest statistically significant upregulation in the chemoresistant tumors. 
Table II. Cancer pathway genes with mRNA levels significantly altered by $>2$-fold in the chemoresistant compared to the chemosensitive ovarian serous carcinomas as determined by the PCR array.

\begin{tabular}{|c|c|c|c|c|c|c|}
\hline Gene symbol & Gene & Function & $\mathrm{CS} / \mathrm{C}$ & $\mathrm{CR} / \mathrm{C}$ & $\mathrm{CR} / \mathrm{CS}$ & P-value \\
\hline \multicolumn{7}{|l|}{ Adhesion } \\
\hline ITGB 1 & Integrin $\beta 1$ & Increase & -7.80 & -3.74 & 2.01 & 0.048 \\
\hline ITGB5 & Integrin $\beta 5$ & Increase & -5.85 & -1.81 & 2.26 & 0.013 \\
\hline \multicolumn{7}{|c|}{ Cell senescence } \\
\hline TERT & Telomerase reverse transcriptase & Increase & 1.62 & 5.21 & 4.23 & 0.001 \\
\hline \multicolumn{7}{|c|}{ Cell cycle control and DNA damage repair } \\
\hline E2F1 & E2F transcription factor 1 & Increase & 9.59 & 20.00 & 2.81 & 0.010 \\
\hline CDKN1A & Cyclin-dependent kinase inhibitor $1 \mathrm{~A}$ & Decrease & -3.45 & -9.09 & -2.56 & 0.006 \\
\hline \multicolumn{7}{|c|}{ Invasion and metastasis } \\
\hline SERPINE1 & Serpin peptidase inhibitor clade E, member 1 & Increase & -331.51 & -30.17 & 9.49 & 0.003 \\
\hline \multicolumn{7}{|c|}{ Signal transduction molecules and transcription factors } \\
\hline FOS & FBJ murine osteosarcoma viral oncogene homolog & Increase & -1.75 & 2.94 & 5.76 & 0.007 \\
\hline
\end{tabular}

CS, chemosensitive; CR, chemoresistant; $\mathrm{C}$, control.

Table III. Apoptosis-related genes with mRNA levels significantly altered by $>2$-fold in the chemoresistant compared to the chemosensitive ovarian serous carcinomas as determined by the PCR array.

\begin{tabular}{|c|c|c|c|c|c|c|}
\hline Gene symbol & Gene & Function & $\mathrm{CS} / \mathrm{C}$ & $\mathrm{CR} / \mathrm{C}$ & $\mathrm{CR} / \mathrm{CS}$ & P-value \\
\hline \multicolumn{7}{|c|}{ TNF ligand, TNFR and TRAF families } \\
\hline FADD & Fas(TNFRSF6)-associated via death domain & Increase & -16.20 & -2.91 & 5.56 & 0.023 \\
\hline TNFRSF10A & TNF receptor superfamily, member $10 \mathrm{~A}$ & Decrease & -2.33 & -5.00 & -2.17 & 0.000 \\
\hline TNFRSF10C & TNF receptor superfamily, member $10 \mathrm{C}$ & Decrease & -1.79 & -11.11 & -6.25 & 0.003 \\
\hline TNFRSF10D & TNF receptor superfamily, member 10D & Decrease & -1.82 & 3.79 & 6.88 & 0.031 \\
\hline TNFRSF1A & TNF receptor superfamily, member $1 \mathrm{~A}$ & Increase & 1.04 & 3.04 & 2.93 & 0.039 \\
\hline TNFRSF8 & TNF receptor superfamily, member 8 & Increase & 1.65 & 5.18 & 3.14 & 0.032 \\
\hline TRAF1 & TNF receptor-associated factor 1 & Decrease & 1.72 & -1.61 & -2.78 & 0.004 \\
\hline
\end{tabular}

CS, chemosensitive; CR, chemoresistant; C, control.

Among 84 genes involved in apoptosis, 4 genes associated with the TNF ligand and TRAF families, FADD (5.56-fold), TNFRSF10D (6.88-fold), TNFRSF1A (2.93-fold) and TNFRSF8 (3.14-fold) were significantly upregulated by $>2$-fold in the chemoresistant compared to the chemosensitive tumors (Table III). FADD, TNFRSF1A and TNFRSF8 mediate apoptotic signals, whereas TNFRSF10D inhibits TRAIL-induced apoptosis. TNFRSF10A, TNFRSF10C and TRAF1 were significantly downregulated by $>2$-fold in this group.

Among 84 genes involved in EMT, 7 genes, FN1 (4.77-fold), GSC (8.15-fold), NOTCH1 (4.45-fold), SMAD2 (2.11-fold), SNAI1 (6.79-fold), SOX10 (5.93-fold) and TMEM132A (3.69-fold) were significantly upregulated by $>2$.0-fold in the chemoresistant group compared to the chemosensitive group (Table IV). GSC, SOX10 and SNAI1 were upregulated by $>5$-fold.
mRNA expression patterns by $q R T-P C R$. qRT-PCR was performed in 60 cases of high-grade serous carcinomas for the genes which were found to be significantly altered in the chemoresistant group in the PCR array (listed in Tables II-IV), and thought to accelerate the oncogenic process. The expression patterns by qRT-PCR were consistent with those of the PCR array. The mean mRNA expression levels of E2F1, FOS, GSC, NOTCH1, SNAI1, SOX10, TERT and TNFRSF10D in the serous carcinomas were higher (7.8-fold, $\mathrm{P}<0.001 ; 3.9$-fold, $\mathrm{P}=0.585 ; 2.5$-fold, $\mathrm{P}=0.865 ; 2.0$-fold, $\mathrm{P}=0.121 ; 2.0$-fold, $\mathrm{P}=0.375 ; 2.2$-fold, $\mathrm{P}=0.003 ; 3.5$-fold $\mathrm{P}=0.129$ and 1.6 -fold, $\mathrm{P}=0.105$, respectively) than those of the control. In contrast, the mean mRNA expression levels of CDKN1A, TNFRSF10A, TNFRSF10C and TRAF1 in the serous carcinomas were lower (0.2-fold, $\mathrm{P}=0.005 ; 0.5$-fold, $\mathrm{P}=0.072 ; 0.3$-fold, $\mathrm{P}=0.003$ and 0.8 -fold, $\mathrm{P}=0.574$, respectively) than those of the control (Fig. 2). 
Table IV. EMT-related genes with mRNA levels by the PCR array significantly altered by $>2$-fold in chemoresistant compared to chemosensitive ovarian serous carcinomas.

\begin{tabular}{lllrrrr}
\hline Gene symbol & \multicolumn{1}{c}{ Gene } & Function & CS/C & CR/C & CR/CS & P-value \\
\hline FN1 & Fibronectin1 & Increase & -26.08 & -5.78 & 4.77 & 0.010 \\
GSC & Goosecoid homeobox & Increase & 3.99 & 30.76 & 8.15 & 0.005 \\
NOTCH 1 & Notch 1 & Increase & 1.00 & 4.22 & 4.45 & 0.004 \\
SMAD2 & SMAD family member 2 & Increase & 1.03 & 2.05 & 2.11 & 0.017 \\
SNAI 1 & Snail homolog 1 & Increase & -1.64 & 3.92 & 6.79 & 0.007 \\
SOX10 & SRY (sex determining region Y)-box 10 & Increase & 4.71 & 26.41 & 5.93 & 0.019 \\
TMEM132A & Transmembrane protein 132A & Increase & -5.68 & -1.63 & 3.69 & 0.005 \\
\hline
\end{tabular}

CS, chemosensitive; CR, chemoresistant; $\mathrm{C}$, control.
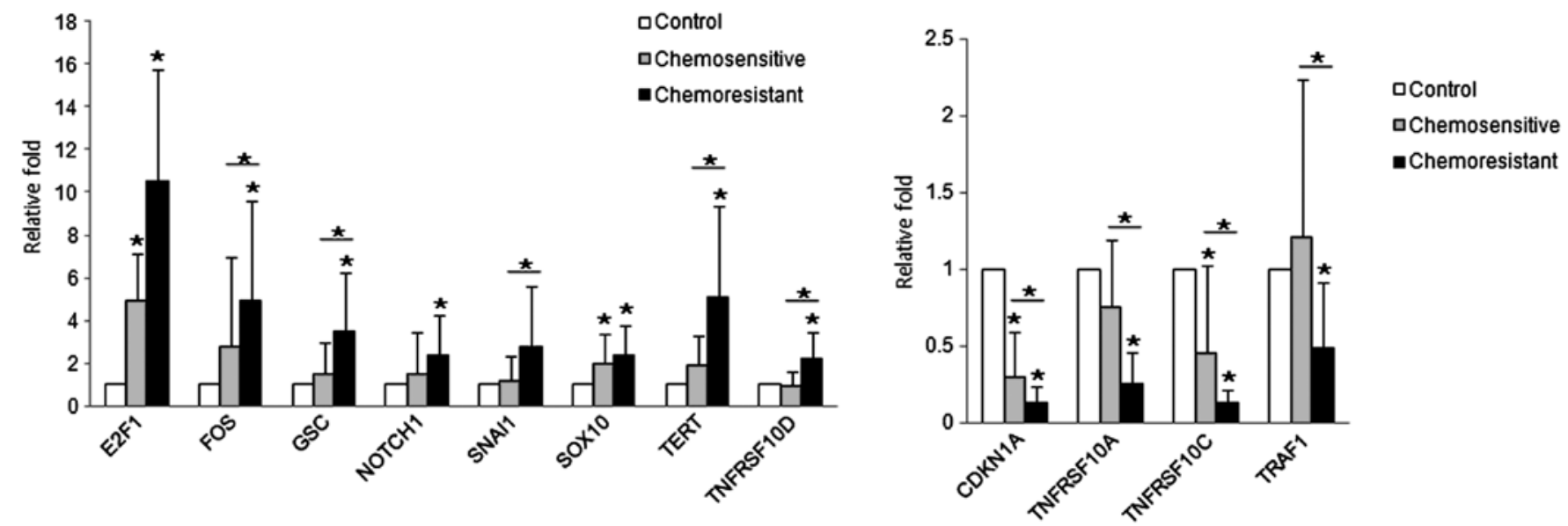

Figure 2. Validation by qRT-PCR for the candidate genes that showed a significantly different mRNA expression between chemosensitive and chemoresistant tumors by PCR array. The bar graph shows the expression of mRNA of each gene in chemosensitive and chemoresistant ovarian serous carcinomas normalized to that of the corresponding GAPDH. Relative expression levels are shown relative to those of the control. The graph represents the mean \pm standard deviation of triplicate experiments ( $\mathrm{P}<0.05)$.

We also analyzed whether the mRNA expression of each gene was significantly different between the chemosensitive and chemoresistant group. Seventeen patients (28.3\%) among the 60 patients with high-grade ovarian serous carcinomas experienced tumor recurrence or persistence after surgery, representing resistance to a first-line chemotherapeutic regimen consisting of Taxol and carboplatin. Among the genes examined, FOS, GSC, SNAI1, TERT and TNFRSF10D showed significantly higher levels (1.8-fold, $\mathrm{P}=0.009 ; 2.2$-fold, $\mathrm{P}=0.021 ; 2.4$-fold, $\mathrm{P}=0.016 ; 2.6$-fold, $\mathrm{P}=0.036 ; 2.4$-fold, $\mathrm{P}=0.006$, respectively) in the chemoresistant group compared with the chemosensitive group. The expression levels of CDKN1A, TNFRSF10A TNFRSF10C and TRAF1 were significantly lower (0.4-fold, $\mathrm{P}=0.006 ; 0.4$-fold, $\mathrm{P}=0.001$; 0.3 -fold, $\mathrm{P}=0.003$ and 0.4 -fold, $\mathrm{P}=0.004$, respectively) than those of the chemosensitive group (Fig. 2).

Correlation of $m R N A$ expression with clinicopathological parameters. The potential association between mRNA expression and clinicopathological parameters, including clinical stage, lymph node metastasis, distant metastasis and survival, was examined to assess the clinical implication of the differentially regulated genes in ovarian serous carcinomas (Table V). Lymph node metastasis was significantly associated with high expression of GSC ( $\geq 1.5$-fold, $\mathrm{P}=0.017$ ) (Fig. 3A). Distant metastasis was significantly associated with low expression of TNFRSF10A $(\leq 0.5$-fold, $\mathrm{P}=0.047)$ and high expression of SNAI1 ( $\geq 1.5$-fold, $\mathrm{P}=0.047$ ) (Fig. 3B). Low expression of TRAF1 ( $\leq 0.5$ fold, $\mathrm{P}=0.036)$ and high expression of $\mathrm{E} 2 \mathrm{~F} 1$ ( $\geq 2$-fold, $\mathrm{P}=0.007$ ), FOS ( $\geq 2$-fold, $\mathrm{P}=0.002$ ), TERT $(\geq 1.5$-fold, $\mathrm{P}=0.015)$ and $\mathrm{GSC}(\geq 1.5$-fold, $\mathrm{P}=0.040)$ were significantly associated with advanced clinical stage (Fig. 3C). The chemoresistance was significantly associated with low expression of TNFRSF10C ( $\leq 0.1$-fold, $\mathrm{P}=0.001)$, TRAF1 $(\leq 0.5$-fold, $\mathrm{P}=0.012$ ) and CDKN1A ( $\leq 0.3$-fold, $\mathrm{P}=0.003)$, and high expression of FOS ( $\geq 2$-fold, $\mathrm{P}=0.012$ ), TERT ( $\geq 1.5$-fold, $\mathrm{P}=0.050$ ), GSC ( $\geq 1.5$-fold, $\mathrm{P}=0.030)$, NOTCH1 $(\geq 1.5$-fold, $\mathrm{P}=0.025)$ and SOX10 ( $\geq 1.5$-fold, $\mathrm{P}=0.024)$.

Survival analysis. The survival analysis was performed for 60 ovarian serous carcinoma patients according to the mRNA expression level of each gene. Follow-up was available for all 
Table V. Correlation between mRNA expression and clinicopathological variables.

\begin{tabular}{|c|c|c|c|c|c|c|c|c|c|c|c|c|}
\hline & \multicolumn{3}{|c|}{$\mathrm{LN}$} & \multicolumn{3}{|c|}{ Metastasis } & \multicolumn{3}{|c|}{ Stage } & \multicolumn{3}{|c|}{ Chemoresponse } \\
\hline & $\mathrm{P}$ & $\mathrm{N}$ & P-value & $\mathrm{P}$ & $\mathrm{N}$ & P-value & III, IV & I, II & $\mathrm{P}$-value & $\mathrm{R}$ & S & P-value \\
\hline \multicolumn{13}{|c|}{ Apoptosis } \\
\hline \multicolumn{13}{|c|}{ TNFRSF10A } \\
\hline$\leq 0.5$ & $12(35.3)$ & $9(34.6)$ & 0.956 & $8(57.1)$ & $13(28.3)$ & $0.047^{\mathrm{a}}$ & $18(38.3)$ & $3(23.1)$ & 0.309 & $8(47.1)$ & $13(30.2)$ & 0.218 \\
\hline$>0.5$ & $22(64.7)$ & $17(65.4)$ & & $6(42.9)$ & $33(71.7)$ & & $29(61.7)$ & $10(76.9)$ & & $9(52.9)$ & $30(69.8)$ & \\
\hline \multicolumn{13}{|c|}{ TNFRSF10C } \\
\hline$\leq 0.1$ & $12(35.3)$ & $10(38.5)$ & 0.801 & $3(21.4)$ & $19(41.3)$ & 0.177 & $18(38.3)$ & $4(30.8)$ & 0.618 & $12(70.6)$ & $10(23.3)$ & $0.001^{\mathrm{a}}$ \\
\hline$>0.1$ & $22(64.7)$ & $16(61.5)$ & & $11(78.6)$ & $27(58.7)$ & & $29(61.7)$ & $9(69.2)$ & & $5(29.4)$ & $33(76.7)$ & \\
\hline \multicolumn{13}{|c|}{ TRAF1 } \\
\hline$\leq 0.5$ & $24(70.6)$ & $14(53.8)$ & 0.182 & $9(64.3)$ & $29(63.0)$ & 0.933 & $33(70.2)$ & $5(38.5)$ & $0.036^{\mathrm{a}}$ & $15(88.2)$ & $23(53.5)$ & $0.012^{\mathrm{a}}$ \\
\hline$>0.5$ & $10(29.4)$ & $12(46.2)$ & & $5(35.7)$ & $17(37.0)$ & & $14(29.8)$ & $8(61.5)$ & & $2(11.8)$ & $20(46.5)$ & \\
\hline \multicolumn{13}{|c|}{ Cancer pathway finder } \\
\hline \multicolumn{13}{|c|}{ CDKN1A } \\
\hline$\leq 0.3$ & $20(58.8)$ & $15(57.7)$ & 0.930 & $9(64.3)$ & $26(56.5)$ & 0.606 & 29 (61.7) & $6(46.2)$ & 0.314 & $15(88.2)$ & $20(46.5)$ & $\mathbf{0 . 0 0 3}^{\mathrm{a}}$ \\
\hline$>0.3$ & $14(41.2)$ & $11(42.3)$ & & $5(35.7)$ & $20(43.5)$ & & $18(38.3)$ & $7(53.8)$ & & $2(11.8)$ & $23(53.5)$ & \\
\hline \multicolumn{13}{|l|}{ E2F1 } \\
\hline$\geq 2$ & $28(82.4)$ & $17(65.4)$ & 0.133 & $11(78.6)$ & $34(73.9)$ & 0.724 & $39(83.0)$ & $6(46.2)$ & $0.007^{\mathrm{a}}$ & $15(88.2)$ & $30(69.8)$ & 0.135 \\
\hline$<2$ & $6(17.6)$ & $9(34.6)$ & & $3(21.4)$ & $12(26.1)$ & & $8(17.0)$ & $7(53.8)$ & & $2(11.8)$ & $13(30.2)$ & \\
\hline \multicolumn{13}{|l|}{ FOS } \\
\hline$\geq 2$ & $18(52.9)$ & $9(34.6)$ & 0.157 & $5(35.7)$ & $22(47.8)$ & 0.425 & $26(55.3)$ & $1(7.7)$ & $0.002^{\mathrm{a}}$ & $12(70.6)$ & $15(34.9)$ & $0.012^{\mathrm{a}}$ \\
\hline$<2$ & $16(47.1)$ & $17(65.4)$ & & $9(64.3)$ & $24(52.2)$ & & $21(44.7)$ & $12(92.3)$ & & $5(29.4)$ & $28(65.1)$ & \\
\hline \multicolumn{13}{|l|}{ TERT } \\
\hline$\geq 1.5$ & $17(50.0)$ & $10(38.5)$ & 0.373 & $8(57.1)$ & $19(41.3)$ & 0.297 & $25(53.2)$ & $2(15.4)$ & $0.015^{\mathrm{a}}$ & $11(64.7)$ & $16(37.2)$ & $0.050^{\mathrm{a}}$ \\
\hline$<1.5$ & $17(50.0)$ & $16(61.5)$ & & $6(42.9)$ & $27(58.7)$ & & $22(46.8)$ & $11(84.6)$ & & $6(35.3)$ & $27(62.8)$ & \\
\hline \multicolumn{13}{|l|}{ EMT } \\
\hline \multicolumn{13}{|l|}{ GSC } \\
\hline$\geq 1.5$ & $21(61.8)$ & $8(30.8)$ & $0.017^{a}$ & $7(50.0)$ & $22(47.8)$ & 0.887 & $26(55.3)$ & $3(23.1)$ & $0.040^{\mathrm{a}}$ & $12(70.6)$ & $17(39.5)$ & $0.030^{\mathrm{a}}$ \\
\hline$<1.5$ & $13(38.2)$ & $18(69.2)$ & & $7(50.0)$ & $24(52.2)$ & & $21(44.7)$ & $10(76.9)$ & & $5(29.4)$ & $26(60.5)$ & \\
\hline \multicolumn{13}{|c|}{ NOTCH1 } \\
\hline$\geq 1.5$ & $13(38.2)$ & $9(34.6)$ & 0.773 & $4(28.6)$ & $18(39.1)$ & 0.473 & $18(38.3)$ & $4(30.8)$ & 0.618 & $10(58.8)$ & 12 (27.9) & $0.025^{\mathrm{a}}$ \\
\hline$<1.5$ & $21(61.8)$ & $17(65.4)$ & & $10(71.4)$ & $28(60.9)$ & & $29(61.7)$ & $9(69.2)$ & & 7 (41.2) & $31(72.1)$ & \\
\hline \multicolumn{13}{|c|}{ SNAI1 } \\
\hline$\geq 1.5$ & $14(41.2)$ & 7 (26.9) & 0.251 & $8(57.1)$ & $13(28.3)$ & $0.047^{\mathrm{a}}$ & $20(42.6)$ & $1(7.7)$ & 0.020 & $9(52.9)$ & $12(27.9)$ & 0.067 \\
\hline$<1.5$ & $20(58.8)$ & $19(73.1)$ & & $6(42.9)$ & $33(71.7)$ & & $27(57.4)$ & $12(92.3)$ & & $8(47.1)$ & $31(72.1)$ & \\
\hline \multicolumn{13}{|c|}{ SOX10 } \\
\hline$\geq 1.5$ & $17(50.0)$ & $15(57.7)$ & 0.554 & $6(42.9)$ & $26(56.5)$ & 0.370 & $25(53.2)$ & $7(53.8)$ & 0.967 & $13(76.5)$ & $19(44.2)$ & $0.024^{a}$ \\
\hline$<1.5$ & $17(50.0)$ & $11(42.3)$ & & $8(57.1)$ & $20(43.5)$ & & $22(46.8)$ & $6(46.2)$ & & $4(23.5)$ & $24(55.8)$ & \\
\hline
\end{tabular}

LN, lymph node. ${ }^{a} \mathrm{P}<0.05, \chi^{2}$-test. $\mathrm{P}$-values indicating statistically significant results are shown in bold print.

57 patients with serous carcinomas, and the mean follow-up period of the study population was 29 months (range, 2-80 months). Nine patients (15.8\%) died of disease during the follow-up period. For each gene, patients were divided into two groups, an upregulation group and a downregulation group, based on the cut-off value of 1.5 -fold (for upregulated genes) or 0.5 -fold (for downregulated genes) compared to the level observed in the control. The upregulation group for TERT (OS:
68.0 vs. $96.9 \%, \mathrm{P}=0.002)$, $\mathrm{GSC}$ (OS 74.1 vs. $93.3 \%, \mathrm{P}=0.039$ ), NOTCH1 (OS: 71.4 vs. 91.7\%, $\mathrm{P}=0.012$ ) and SANI1 (OS: 61.9 vs. $97.2 \%, \mathrm{P}=0.001$ ) demonstrated a statistically significant decrease in overall survival (Fig. 4) compared with that of the downregulation group. The downregulation group for TRAF1 (OS: 75.0 vs. $100.0 \%, \mathrm{P}=0.008$ ) demonstrated a statistically significant decrease in overall survival (Fig. 4) compared with that of the upregulation group. 
A

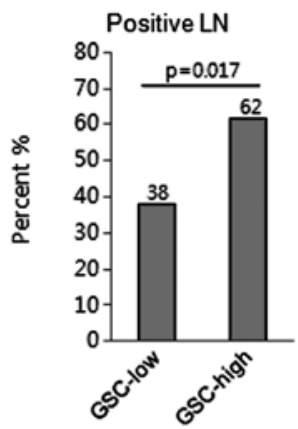

B

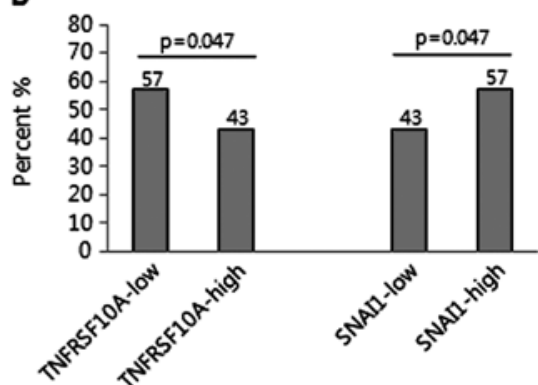

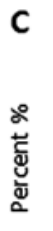

C

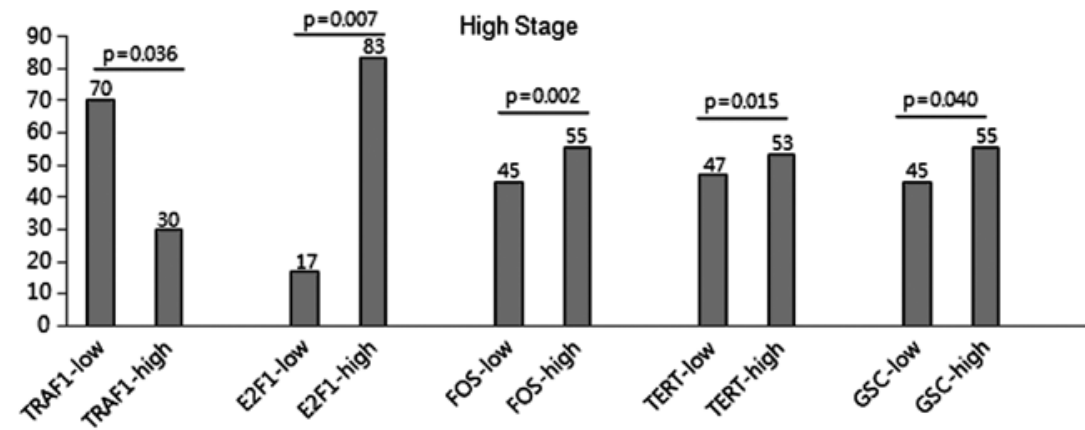

Figure 3. Correlation of mRNA expression by qRT-PCR with clinicopathological parameters. (A) High expression of GSC ( $\geq 1.5$-fold) was significantly associated with LN metastasis. (B) Low expression of TNFRSF10A ( $\leq 0.5$-fold) and high expression of SNAI1 ( $\geq 1.5$-fold) were significantly associated with distant metastasis. (C) Low expression of TRAF1 ( $\leq 0.5$-fold) and high expression of E2F1 ( $\geq 2$-fold), FOS $(\geq 2$-fold), TERT ( $\geq 1.5$-fold) and GSC $(\geq 1.5$-fold) were significantly associated with advanced clinical stage (stages III and IV).
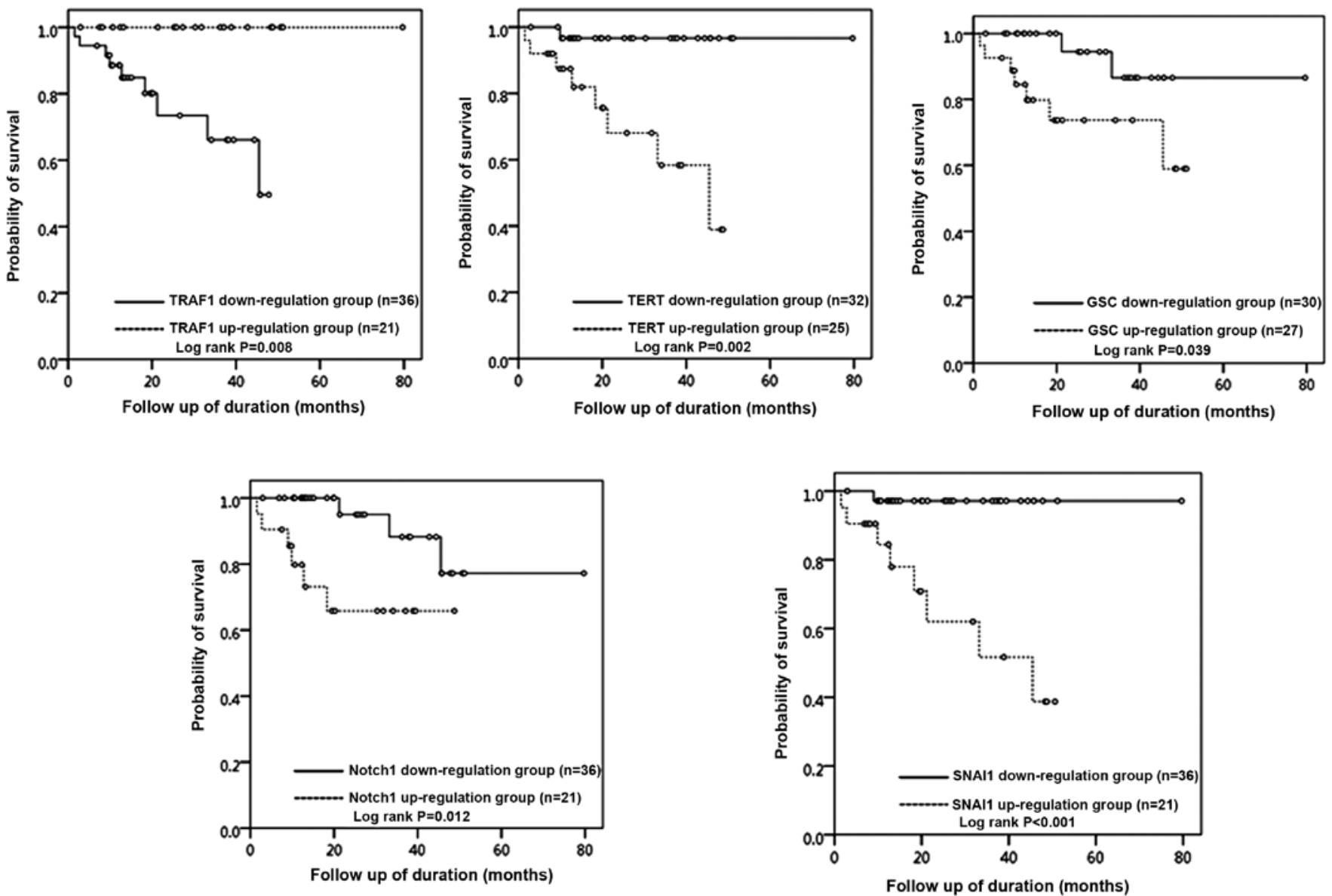

Figure 4. Kaplan-Meier overall survival curves. The downregulation group of TRAF1 ( $\leq 0.5$-fold) and upregulation group ( $\geq 1.5$-fold) of TERT, GSC, NOTCH1 and SNAI1 were associated with a significantly worse overall survival than the upregulation or downregulation group, respectively, in the ovarian serous carcinoma cases. 
A
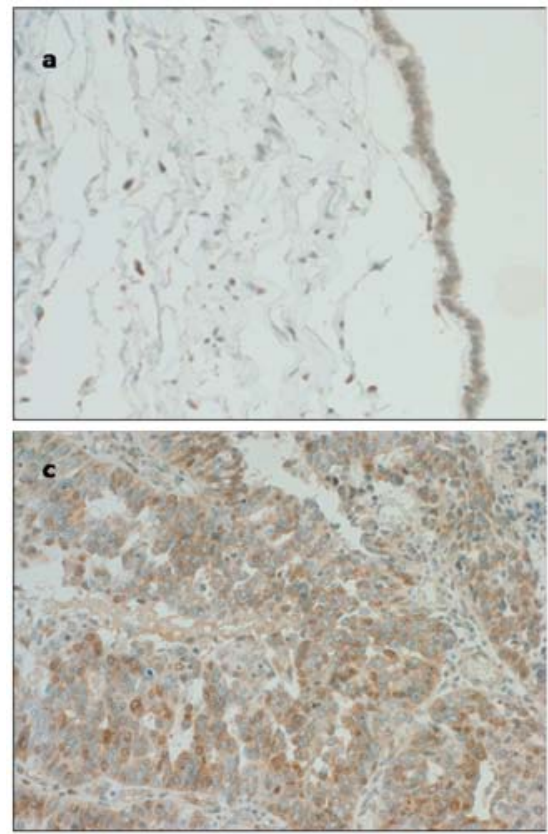

B

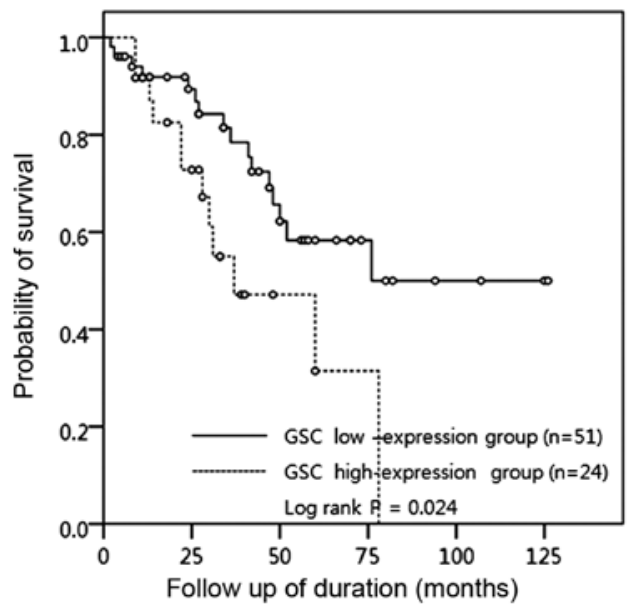

Validation with TMA and immunohistochemistry. We performed immunohistochemistry with a larger number of 75 ovarian serous carcinomas for the genes which showed a significant association of mRNA expression with more than 2 clinicopathological parameters and survival, such as TRAF1, TERT and GSC to validate their clinical impact at the protein level. Among the proteins examined, GSC overexpression showed a significant association with patient survival. The nuclear and/or cytoplasmic expression of GSC protein was observed in 48 (64\%) of 75 ovarian serous carcinomas, whereas it was weakly expressed in $<10 \%$ of epithelial cells of normal fallopian tubes or benign serous tumors. The overexpression of GSC (positive cells $>50 \%$ of tumor cells) was found in 24 (32\%) ovarian serous carcinomas (Fig. 5A). Among 75 patients, 28 patients $(37.3 \%)$ died of disease during the follow-up period. A Kaplan-Meier survival analysis (Fig. 5B) revealed that the GSC-overexpressing group was significantly correlated with poor patient survival (OS: 50.0 vs. 68.6\%, $\mathrm{P}=0.024)$. The expression of TRAF1 or TERT did not show a significant difference between the control and the malignant serous tumors, and any association with patient survival.

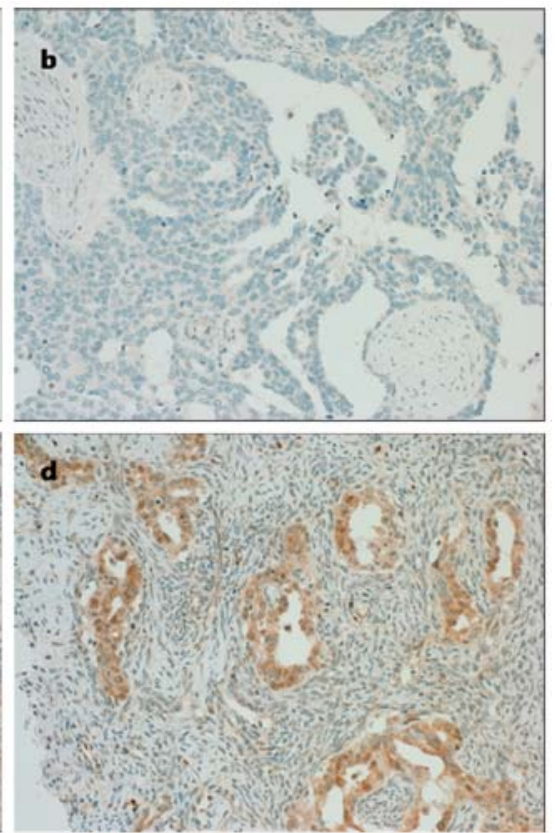

Figure 5. (A) Representative images of immunohistochemical staining for the GSC protein. (a) Serous cystadenoma showing $<10 \%$ positivity for the GSC protein. (b) Serous adenocarcinoma showing negativity for the GSC protein (original magnification, x200). (c and d) Serous adenocarcinoma showing overexpression ( $>50 \%$ positivity) of the GSC protein (original magnification, x200). (B) Kaplan-Meier overall survival curve. The high GSC-expressing group ( $>50 \%$ positivity in tumor cells) upon immunohistochemical staining was associated with a significantly worse overall survival than the low expressing group in the ovarian serous carcinoma cases.

\section{Discussion}

Ovarian carcinoma is the most frequent cause of mortality among gynecological malignancies. Treatment generally consists of surgical cytoreduction and platinum/taxane-based chemotherapy (13). The majority of patients with advanced stage eventually relapse within 18 months, many with chemoresistance. Indeed, $90 \%$ of the deaths from ovarian cancer may be attributed to chemoresistance (5). Therefore, the development of strategies to overcome chemoresistance in ovarian cancer is urgently needed.

Recent studies indicate that chemoresistance is multifactorial and may involve increased drug inactivation/efflux, increased DNA repair, alterations in cell cycle control and changes in apoptosis. Several signal transduction pathways, including PI3K/AKT and MAPK, are involved in the drug resistance in many malignancies $(8,14)$. A better understanding of the molecular mechanism of chemoresistance may lead to new approaches to overcome it and improve survival; however, despite vigorous efforts, no marked advances have been reported in the clinical field. 
In the present study, to identify genes contributing to the development of chemoresistance, the gene expression of ovarian carcinomas was profiled by PCR arrays, and chemoresistant tumors were compared to chemosensitive tumors. The selected arrays focused on apoptosis, EMT and cancer development pathways since the response to chemotherapeutic agents is mainly dependent upon apoptotic ability, and since chemoresistance is associated with EMT $(15,16)$. We then validated mRNA expression by qRT-PCR for the candidate genes, which showed a significantly differential expression between chemosensitive and chemoresistant tumors.

For cancer development pathways, the qRT-PCR arrays contained 84 genes involved in cell adhesion, senescence, cell cycle control, invasion/metastasis, apoptosis and signal transduction/transcription. Six genes, including ITGB1, ITGB5, TERT, E2F1, SERPINE1 and FOS, were upregulated by $>2$-fold in the chemoresistant compared to the chemosensitive tumors. However, ITGB1, ITGB5 and SERPINE1 were unexpectedly downregulated in the cancer tissues compared to normal epithelium; therefore, these genes were not thought to have a significant impact on oncogenesis or chemoresistance in this subset of ovarian carcinomas. On the other hand, upregulation of TERT and FOS was associated with advanced clinical stage as well as chemoresistance. In terms of apoptosis, four genes associated with the TNF ligand and TRAF families, FADD, TNFRSF10D, TNFRSF1A and TNFRSF8, were significantly upregulated by $>2$-fold in the chemoresistant compared to the chemosensitive tumors. The other apoptotic genes, such as TNFRSF10A, TNFRSF10C and TRAF1, were significantly downregulated by $>2$-fold in the chemoresistant compared to the chemosensitive group. Since FADD, TNFRSF1A and TNFRSF8 mediate apoptosis, upregulation of these genes in the chemoresistant tumors was an unexpected finding; however, when taking into consideration that TNFRSF1A and TFFRSF8 also activate the NF- $\mathrm{KB}$ pathway, and an anti-apoptotic gene, TNFRSF10D, was highly upregulated, the overall apoptotic balance was likely to favor decreased apoptosis in the chemoresistant group of tumors.

Emerging evidence suggests that EMT is associated with chemoresistance (10) and with the acquisition of the cancer stem cell phenotype. EMT is a developmental process that plays an important role in tumor progression and metastasis in many cancers, including ovarian cancer (17). Our results showed that typical EMT-inducing genes, including FN1, GSC, NOTCH1, SMAD2, SNAI1, SOX10 and TMEM132A, were significantly upregulated in the chemoresistant tumors. However, FN1 and TMEM132A were downregulated in the cancer tissues compared to normal epithelium, suggesting that these genes did not play important roles in oncogenesis in this tumor. The other genes, such as GSC, NOTCH1, SNAI1 and SOX 10, showed a significant association with poor prognostic factors as well as with chemoresistance by qRT-PCR in the present study.

When assessing the correlation between these genes and clinicopathological variables, low expression of TRAF1 and CDKN1A, and high expression of FOS, TERT and GSC were significantly associated with advanced clinical stage. Lymph node metastasis was significantly associated with high expression of GSC, and distant metastasis was significantly associated with low expression of TNFRSF10A and high expression of SNAI1, implying that these genes are implicated in tumor progression.

Among the genes with significantly altered mRNA expression in the chemoresistant tumors, those which showed a significant association with more than 2 clinicopathological parameters and survival, including TRAF1, TERT and GSC, were selected for verification at the protein level. The differences in protein levels reached statistical significance only for GSC protein, whereas the expression of TRAF1 or TERT did not show a significant difference between the benign and malignant serous tumors. When we further analyzed the impact of these proteins on prognostic factors and survival, high expression of GSC protein was associated with poor overall survival upon immunohistochemical analysis, suggesting that GSC is the most predictive and prognostic biomarker for the chemoresistance and patient overall survival in ovarian serous carcinoma.

GSC is a homeobox-containing protein first reported as a transcriptional repressor regulating formation and patterning in vertebrate embryos (18-20). Notably, elements of the TGF- $\beta$ superfamily and Wnt/ $\beta$-catenin signaling pathways, which are involved in tumor invasion and metastasis through EMT $(21,22)$, can induce GSC expression in embryonic cells $(23,24)$. Therefore, GSC is anticipated to play a role in neoplastic disease. A recent study found that GSC is overexpressed in human breast carcinomas and plays an important role in activating cell properties associated with tumor progression to malignancy and metastasis in breast cancer cells (25). In accordance with their study, we demonstrated that GSC is significantly correlated with poor prognostic factors, such as advanced clinical stage and lymph node metastasis, and poor overall survival in high-grade ovarian serous carcinomas. This is the first report that GSC is implicated in chemoresistance and poor survival in human cancer, suggesting that GSC is a potential biomarker of drug response or novel therapeutic strategies for overcoming drug resistance in ovarian serous carcinomas. Further study with a large scale of clinical samples and functional study on chemoresistant ovarian cancer cells may support our suggestion.

\section{Acknowledgements}

This research was supported by the Basic Science Research Program of the National Research Foundation of Korea (NRF) funded by the Ministry of Education, Science and Technology (NRF-2012-R1A1B3004095).

\section{References}

1. Jemal A, Thomas A, Murray T and Thun M: Cancer statistics, 2002. CA Cancer J Clin 52: 23-47, 2002.

2. McGuire WP, Hoskins WJ, Brady MF, et al: Cyclophosphamide and cisplatin compared with paclitaxel and cisplatin in patients with stage III and stage IV ovarian cancer. New Engl J Med 334: $1-6,1996$

3. Chan WY, Cheung KK, Schorge JO, et al: $\mathrm{Bcl}-2$ and $\mathrm{p} 53$ protein expression, apoptosis, and p53 mutation in human epithelial ovarian cancers. Am J Pathol 156: 409-417, 2000.

4. Shih Ie M and Kurman RJ: Ovarian tumorigenesis: a proposed model based on morphological and molecular genetic analysis. Am J Pathol 164: 1511-1518, 2004.

5. Agarwal R and Kaye SB: Ovarian cancer: strategies for overcoming resistance to chemotherapy. Nat Rev Cancer 3: 502-516, 2003. 
6. Shahzad MM,Lopez-Berestein G and Sood AK: Novel strategies for reversing platinum resistance. Drug Resist Updat 12: 148-152, 2009.

7. Stordal B, Pavlakis N and Davey R: A systematic review of platinum and taxane resistance from bench to clinic: an inverse relationship. Cancer Treat Rev 33: 688-703, 2007.

8. Garcia-Echeverria C and Sellers WR: Drug discovery approaches targeting the PI3K/Akt pathway in cancer. Oncogene 27: 5511-5526, 2008.

9. Fan F, Samuel S, Evans KW, et al: Overexpression of snail induces epithelial-mesenchymal transition and a cancer stem cell-like phenotype in human colorectal cancer cells. Cancer Med 1: 5-16, 2012.

10. Wang Z, Li Y, Kong D, et al: Acquisition of epithelial-mesenchymal transition phenotype of gemcitabine-resistant pancreatic cancer cells is linked with activation of the notch signaling pathway. Cancer Res 69: 2400-2407, 2009.

11. Haslehurst AM, Koti M, Dharsee M, et al: EMT transcription factors snail and slug directly contribute to cisplatin resistance in ovarian cancer. BMC Cancer 12: 91, 2012.

12. Kurrey NK, Amit K and Bapat SA: Snail and Slug are major determinants of ovarian cancer invasiveness at the transcription level. Gynecol Oncol 97: 155-165, 2005.

13. Cannistra SA: Cancer of the ovary. N Engl J Med 351: 2519-2529, 2004.

14. Wu H, Cao Y, Weng D, et al: Effect of tumor suppressor gene PTEN on the resistance to cisplatin in human ovarian cancer cell lines and related mechanisms. Cancer Lett 271: 260-271, 2008.

15. Marchini S, Fruscio R, Clivio L, et al: Resistance to platinum-based chemotherapy is associated with epithelial to mesenchymal transition in epithelial ovarian cancer. Eur J Cancer 49: 520-530, 2012.
16. Chen X, Lingala S, Khoobyari S, Nolta J, Zern MA and Wu J: Epithelial mesenchymal transition and hedgehog signaling activation are associated with chemoresistance and invasion of hepatoma subpopulations. J Hepatol 55: 838-845, 2011.

17. Ahmed N,Thompson EW and Quinn MA:Epithelial-mesenchymal interconversions in normal ovarian surface epithelium and ovarian carcinomas: an exception to the norm. J Cell Physiol 213: 581-588, 2007.

18. Niehrs C, Keller R, Cho KW and De Robertis EM: The homeobox gene goosecoid controls cell migration in Xenopus embryos. Cell 72: 491-503, 1993.

19. Blumberg B, Wright CV, De Robertis EM and Cho KW: Organizer-specific homeobox genes in Xenopus laevis embryos. Science 253: 194-196, 1991.

20. Blum M, Gaunt SJ, Cho KW, et al: Gastrulation in the mouse: the role of the homeobox gene goosecoid. Cell 69: 1097-1106, 1992.

21. Thiery JP: Epithelial-mesenchymal transitions in tumour progression. Nat Rev Cancer 2: 442-454, 2002.

22. Grunert S, Jechlinger $M$ and Beug H: Diverse cellular and molecular mechanisms contribute to epithelial plasticity and metastasis. Nat Rev Mol Cell Biol 4: 657-665, 2003.

23. Moon RT and Kimelman D: From cortical rotation to organizer gene expression: toward a molecular explanation of axis specification in Xenopus. BioEssays: 20: 536-545, 1998.

24. Watabe T, Kim S, Candia A, et al: Molecular mechanisms of Spemann's organizer formation: conserved growth factor synergy between Xenopus and mouse. Genes Dev 9: 3038-3050, 1995.

25. Hartwell KA, Muir B, Reinhardt F, Carpenter AE, Sgroi DC and Weinberg RA: The Spemann organizer gene, Goosecoid, promotes tumor metastasis. Proc Natl Acad Sci USA 103: 18969-18974, 2006. 